\title{
Philosophiques
}

\section{Sens et vérité chez Clauberg et Spinoza}

\section{Jacqueline Lagrée}

Volume 29, numéro 1, printemps 2002

Spinoza sous le prisme de son anthropologie

URI : https://id.erudit.org/iderudit/009568ar

DOI : https://doi.org/10.7202/009568ar

Aller au sommaire du numéro

Éditeur(s)

Société de philosophie du Québec

ISSN

0316-2923 (imprimé)

1492-1391 (numérique)

Découvrir la revue

Citer cet article

Lagrée, J. (2002). Sens et vérité chez Clauberg et Spinoza. Philosophiques, 29(1), 121-138. https://doi.org/10.7202/009568ar d'utilisation que vous pouvez consulter en ligne.

https://apropos.erudit.org/fr/usagers/politique-dutilisation/ 


\title{
Sens et vérité chez Clauberg et Spinoza
}

\section{JACQUELINE LAGRÉE}

UFR de philosophie, Université de Rennes I

Jacqueline.Lagree@univ-rennesı.fr

\begin{abstract}
RÉSUMÉ. - L'article montre que la position de Spinoza dans le Traité théologicopolitique s'enracine dans une tradition herméneutique marquée par la Logique de J. Clauberg qui distingue entre le sens authentiquement visé par l'auteur (sensus genuinus), le sens vrai et la vérité. Cette distinction est reprise par L. Meyer, le médecin ami de Spinoza dans l'Interpres, en réduisant le sens vrai à la vérité comprise par la philosophie de Descartes ou Spinoza. Spinoza en revanche maintient la distinction entre vérité et sens vrai, en conservant à l'Écriture sainte son caractère sacré dès lors qu'elle facilite la pratique de la justice et de la charité. La raison dégage les enseignements moraux universels de la Bible en favorisant une communauté dans la recherche du vrai. Ainsi peut-il soutenir un principe d'adaptation large du lecteur dans sa lecture de la Bible et défendre une herméneutique tolérante et généreuse sans jamais rien céder de la rigueur de l'interprétation.
\end{abstract}

\begin{abstract}
This paper aims to show how the Spinozistic hermeneutical position in the Tractatus Theologico-Politicus is founded in a particular tradition, that of ). Clauberg, which makes a strong distinction among the sensus genuinus (i.e., the meaning intended by the author), the true meaning, and the truth. L. Meyer, a physician and friend of Spinoza, accepts this distinction but he reduces the true meaning to the truth as it is understood in the philosophy of Descartes or Spinoza. Spinoza, however, maintains the distinction between true meaning and truth, and for him the Bible keeps its holy character as long as it helps man to practice justice and charity. Human reason brings out the universal moral teachings of the Bible and it fosters a community of those who genuinely seek the truth. Thus can Spinoza support a wide principle of adaptation of the reader towards the Bible and defend a tolerant and generous hermeneutic without ever abandoning the rigour of true interpretation.
\end{abstract}

Celui qui agit parfaitement est semblable [...] à un savant auteur qui renferme le plus de réalité dans le moins de volume qu'il peut. ${ }^{1}$

La question de l'herméneutique est une question aussi ancienne que l'existence de textes de référence susceptibles d'être interprétés. Quand Spinoza s'insère dans cette querelle, il ne part pas de rien mais de discussions dont la plupart nous sont devenues obsolètes, voire inintelligibles. Les hommes de l'âge classique ont déjà conscience d'une sorte d'inflation de la demande herméneutique. Si les Anciens en éprouvaient un moindre besoin que les Modernes, c'est qu'ils disposaient de moins de documents écrits et que la théologie et la jurisprudence y étaient moins développées ${ }^{2}$. Aussi les logiques du XVII

1. Leibniz, Discours de métaphysique $\$ 5$.

2. Prol $\$ 124$ Fatemur interim Hermeneuticam illam analyticam non fuisse omni aevo aeque necessariam. Nam Veteres apud quos aut nulla aut pauca admodum exstabant 
intègrent-elles un art de l'interprétation correcte bien distingué de la grammaire et de la rhétorique comme on le voit tant chez Arnauld et Nicole que chez Johannes Clauberg, disciple et défenseur fervent de Descartes ${ }^{3}$.

Les progrès de l'exégèse scripturaire aussi bien que de la connaissance de l'histoire des manuscrits, tant profanes que sacrés, de leurs variantes et des différentes leçons ${ }^{4}$, avaient déjà conduit à distinguer entre le sens authentiquement visé par l'auteur (sensus genuinus, sensus verus) et le sens qui correspond à la vérité de la chose dont il est question. En outre, la vénération pour certains auteurs du passé (Aristote, Platon ou Thomas) jointe au sentiment d'un bouleversement légitime et irréversible du paysage scientifique et philosophique, avec l'avènement de la nouvelle philosophie et de la nouvelle physique, imposait de dégager le sens authentique du sens reçu, de débarrasser ce dernier des gangues d'interprétations successives afin de rendre éventuellement possible un accord renouvelé entre Anciens et Modernes. Enfin, depuis le début de la Réforme, les débats exégétiques sur l'Écriture Sainte, sur sa clarté ou son obscurité, la détermination de son interprète légitime, la délimitation et la vérité de son message, avaient renouvelé l'intérêt pour des analyses comme celles d'Aristote dans le Péri herménéias ou d'Augustin dans le De doctrina christiana.

Clauberg, tout comme les cartésiens qui prennent la mathématique comme modèle du savoir, insiste très fortement sur l'omnitemporalité du vrai. Le vrai n'est ni ancien ni nouveau « Rien n'est plus ancien que la vérité » écrivait Descartes dans sa lettre préface aux Doyens de Sorbonne ${ }^{5}$ et Clauberg de renchérir :

Car il convient d'embrasser l'ancien non en tant qu'ancien ou le nouveau non en tant que nouveau, mais parce que cela saisit le vrai. Et ce qui, un jour, est vrai de la nature de quelque chose, est toujours vrai d'elle, soit que cela n'ait été que récemment connu des hommes, soit que cela ait été manifeste depuis longtemps ${ }^{6}$.

exstabant monumenta scripta, minus ea indigebant. Quae causa est cur illis non fuerit tradita et ab Aristotele in lib. de Interpretatione vix primis lineamentis adumbrata.

3. Né à Solingen en Westphalie en 1622, calviniste, Clauberg fit des études de philosophie et de théologie à Brême puis à Groningue où il se lia d'amitié avec Tobie André qui l'initia à Descartes. Il fit le traditionnel voyage des Réformés en France, notamment à Saumur ; il y lia amitié avec Clerselier, de la Forge, le chancelier Seguier ; puis il alla à Leyden se perfectionner dans la physique de Descartes auprès de De Raey. Il obtint la chaire de philosophie à Herborn avant d'enseigner la philosophie à Duisburg dont il fut le premier recteur ; il mourut en 1665.

4. Voir par exemple la découverte du caractère tardif de la vocalisation de l'Écriture Sainte par les Massorètes ou la découverte par Casaubon de la datation post chrétienne du Corpus hermétique.

5. Descartes, Lettre à Mrs les Doyens de Sorbonne, quia nibil est veritate antiquius, AT VII 3, 25 et AT IX, 6 pour la trad. fr.

6. [...] cum antiqua non quia antiqua neque nova quia nova sed quod vera deprehensa amplecti solum deceat. Et quod aliquando verum est de alicujus rei natura semper de ea verum est sive nuper hominibus innotuerit, sive diu fuerit perspectum. Prol. $\$ 93$ dans l'édition de 1658 ; IV, xi, $\$ 97$ dans l'édition de 1654. 
Dans la pléiade des cartésiens, pourquoi privilégier Clauberg ? Pas seulement pour sa définition de l'ontologie dans l'Ontosophia ni pour sa détermination de l'étant comme cogitabile mais bien ici pour son influence incontestable sur L. Meyer et par lui sur Spinoza ${ }^{7}$. Lorsque Spinoza écrit le Traité théologico-politique, Meyer a déjà publié anonymement La philosophie interprète de l'Ecriture sainte. Or entre le Traité théologico-politique et l'Interpres, il y a une opposition nette quant au statut de la vérité dans le texte sacré. Pour le comprendre, il faut faire le détour claubergien. La logique, telle qu'on la conçoit au XVII ${ }^{\mathrm{e}}$ siècle, n'est pas seulement un art de raisonner de façon correcte, c'est un art de penser ${ }^{8}$ dont les enjeux intersubjectifs - se faire comprendre d'autrui et le comprendre — ont immédiatement des conséquences pratiques, politiques, éthiques et théologiques. Pour se former comme pour se transmettre, la pensée doit s'extérioriser pour quelqu'un, selon sa capacité, dans une situation donnée qui détermine la forme du discours adapté (récit, démonstration, questions et réponses, métaphore...) et cette extériorisation inaugure un processus ouvert d'explicitation, d'interprétation, de réfutation, marqué par les aléas et les progrès de la démarche intellectuelle, dans un rapport à autrui qui s'inscrit à la fois dans la durée et dans un contexte linguistique et culturel donné.

Tout le débat sur l'interprétation de l'Écriture Sainte au XVII siècle, de son interprète et de sa norme, passe par la détermination de la distinction entre sens authentiquement visé (sensus genuinus), sens vrai (sensus verus) et vérité. Il ne suffit pas de déterminer si tel passage doit être compris au sens littéral ou en un sens figuré et à quel niveau de sens figuré ${ }^{9}$, il faut aussi se demander quel était le sens visé par le rédacteur et si celui-ci parlait en son nom propre ou n'était que le secrétaire (amanuensis), le porte plume, le " notaire ${ }^{10}$, du seul véritable auteur, Dieu.

Pour prendre un exemple emprunté à la Logique de Port Royal ${ }^{11}$, si l'on dit d'un prince qu'il a été zélé pour la véritable religion, le sens authentiquement visé par l'historien hagiographe est clair : la véritable religion,

7. Spinoza possédait sa Logique dans sa bibliothèque: Catalogue $\mathrm{n}^{\circ} 29$, édition d'Amsterdam, 1654. Spinoza disposait également de la Defensio cartesiana de $1652\left(\mathrm{n}^{\circ} 28 \mathrm{du}\right.$ catalogue).

8. Cf. «Il est vrai que l'on eût pu dire l'art de bien penser ; mais cette addition n'était pas nécessaire, étant assez marquée par le mot d'art qui signifie de soi-même une méthode de bien faire quelque chose comme Aristote même le remarque "Logique de Port Royal, II Discours.

9. Suivant la théorie médiévale des quatre sens : sens littéral, sens allégorique, sens moral, sens anagogique. Par ex. le bras de Dieu ne désigne pas un membre corporel (sens littéral) mais la puissance divine (sens allégorique) ; l'alliance avec Abraham préfigure l'alliance avec le Christ (sens anagogique).

10. Terme employé par Calvin (notaire juré du saint Esprit) pour désigner les rédacteurs de livres saints.

11. La Logique ou l'art de penser, 1662, citée LPR dans l'édition Clair Girbal, PUF, 1965. 
c'est-à-dire au sens propre ou vrai celle qui est conforme à ce que Dieu attend des hommes et donc celle qui procure le salut, est celle qu'on pratique au royaume de ce Prince ${ }^{12}$.

Et si on lit dans un historien qu'un prince a été zélé pour la véritable religion on ne saurait dire ce qu'il a entendu par là si on ne sait de quelle religion a été cet historien : car si c'est un protestant, cela voudra dire la religion protestante ; si c'était un arabe mahométan qui parlât ainsi de son prince cela voudrait dire la religion mahométane et on ne pourrait juger que ce serait la religion catholique si on ne savait que cet historien est catholique ${ }^{13}$.

Pour Arnauld, de toute évidence, si l'historien est protestant, le sens authentique est que cette religion est la religion dite réformée ${ }^{14}$ mais ce sens véritablement visé ne correspond pas au sens vrai puisque la seule véritable religion est le christianisme sous sa forme catholique. "Lorsque l'esprit a joint cette idée de véritable religion à une idée distincte d'un certain culte particulier distinctement connu, ce mot devient très équivoque et signifie dans la bouche de chaque peuple le culte qu'il prend pour véritable. ${ }^{15}$

On se demandera de quelle discipline relèvent ces distinctions. De la grammaire ? De la rhétorique ? Ce sont des arts subordonnés, auxiliaires de la logique. C'est l'usage qui est le maître de la formation des mots et de leur sens littéral. Dans la conversation ordinaire l'usage suffit mais il n'en va pas exactement de même lorsqu'il s'agit de l'art d'user de sa raison. Même si le bon sens est chose commune, son usage correct n'est pas si fréquent. Nous parlerons correctement si nous suivons l'usage commun de parler mais nous ne raisonnerons pas correctement si nous imitons la manière commune de se servir de la raison. ${ }^{16} \mathrm{La}$ langue est entièrement et légitimement régie par l'usage, le discours par la logique comme art de penser rigoureusement. La logique a ainsi une vertu thérapeutique face aux maladies de l'âme que sont l'erreur, l'ignorance, l'obscurité, la confusion, la fausseté du jugement, l'inconséquence. ${ }^{17} \mathrm{Il}$ serait assez facile de dresser ici un parallèle avec la fin du prologue du TIE (\$17-18) de Spinoza où la guérison de la maladie quasi mortelle du déchirement de l'âme $(\$ 1-11)$ est suivie de l'énoncé de quelques règles de vie bonne qui fonctionnent comme des principes de diététique.

12. Cf. le principe Cujus regio, cujus religio.

13. LPR I, 8, p. 67.

14. Qu'Arnauld, lui, appelle la « religion prétendue réformée ".

15. Ibid, p. 69.

16. Si usum loquendi vulgarem sequamur, recte loquemur; at non recte ratiocinabimur si vulgarem ratione utendi modum imitemur Prol. $\$ 99$.

17. Prol. $\$ 3$. 


\section{La conception herméneutique de la Logique ${ }^{18}$ de Clauberg}

La logique de Clauberg n'est pas une logique au sens moderne. Elle intègre une dimension thérapeutique - soigner les maladies de l'âme - et une dimension éthique - permettre une meilleure compréhension entre les hommes, distinguer entre interprétation vraie et calomnie ${ }^{19}$ - parce que, dans ce type de défaillances, c'est le sujet malade qui doit être son propre médecin : mens sibi ipsi medicus esse debet ${ }^{20}$. L'art de la logique ayant été inventé tout autant pour bien conduire l'esprit humain dans la formation de ses pensées que pour la compréhension ou résolution (analysis) correcte de celle d'autrui, la logique sera quadripartite : une première division concerne la distinction entre art de bien former ses pensées et celui de bien les exprimer à autrui, la seconde distingue entre l'art d'interpréter le discours d'autrui et l'art de bien juger de la vérité de son discours.

\subsection{Définition de la logique herméneutique}

L'herméneutique, art d'interpréter le sens, se retrouve dans les deux divisions de la logique : comme herméneutique génétique — ou art de faire passer à autrui le sens qu'on veut signifier, art d'enseigner donc - et comme herméneutique analytique ou art de déchiffrer ce qu'autrui a voulu dire, art de comprendre. Cette dernière cherche à comprendre ce qu'a vraiment voulu dire autrui ; c'est proprement l'art du bonus interpres, particulièrement utile aujourd'hui ${ }^{21}$, aux jurisconsultes et aux théologiens, en raison de la multiplication des textes qui font autorité. S'il existe bien des règles d'interprétation du sens vrai propres à chaque discipline ${ }^{22}$, la logique porte sur celles qui sont communes à tous les types de discours. Pour autant elle ne se confond pas avec l'ontologie ${ }^{23}$ qui porte sur les attributs communs des étants, quae proprie ad scientiam entis quatenus entis pertinet. Enfin, le fait que l'herméneutique génétique précède l'herméneutique analytique signifie que le rapport au vrai et au discours est centré sur l'ego (première vérité de la métaphysique) et non pas sur l'expérience, c'est-à-dire à autre chose que l'ego (qu'il s'agisse des données sensibles ou du discours d'autrui).

18. L'édition définitive de 1658 se distingue de la première édition de 1654 par de longs prolégomènes $(124 \$)$ qui portent sur les causes psychologiques et sociales d'erreurs (les préjugés de l'enfance, la précipitation et la prévention, la conversation en société) à partir de quoi est posée la nécessité de recourir à la logique. Sauf indication contraire, nous citerons dans l'édition de 1658.

19. L'opposition n'est pas ici entre un bon et un mauvais interprète au sens de savant et ignorant (comme il y a de bons et de mauvais traducteurs) mais entre le bonus interpres et le Calumniator et Sycophanta malitiosus (Prol. \$124).

20. Prol. $\$ 100$.

21. Prol. $\mathbb{S} 123-124$.

22. Comme la philologie et la grammaire des langues anciennes pour l'Écriture Sainte, par exemple.

23. I, vi, $\$ 43$, Ontosophia dans l'édition de 1654. 


\subsubsection{Sens vrai et vérité}

Clauberg soutient, comme à la même époque Port Royal et la majorité des cartésiens, une conception représentative du signe liée à la détermination représentative de l'idée ${ }^{24}$. La signification est comme la forme ou l'âme du mot : significatio est forma et velut anima vocis ${ }^{25}$. Le sens est ce qui est conçu par l'esprit (conceptus mentis ou jugement complexe). Il correspond à l'usage courant de la langue mais on doit éventuellement distinguer entre un sens usuel et un sens savant. Par exemple, la question an detur vacuum ? n'a pas le même sens si on évoque une piscine vidée d'eau ou un bateau vidé de sa cargaison ou si on la prend au sens des physiciens où le vide désigne un espace où il n'y a aucune substance corporelle ${ }^{26}$. Le sens est donné par la définition, définition de chose quand le terme est univoque, définition de nom quand on le prend en un sens propre à une discipline. Par exemple justitia est constans et perpetua voluntas jus suum cuique tribuendi ${ }^{27}$.

Si l'art d'exprimer adéquatement ses pensées appartient à la logique, l'art d'interpréter en relève aussi. Clauberg produit ici une distinction fine, amplement reprise ensuite dans les débats sur l'interprétation de l'Écriture : le sensus genuinus (ou germanus et mentis dicentis congruus) le sens authentique, est le sens visé effectivement par le locuteur. C'est ce sens qu'il faut rechercher d'abord, sans se demander s'il est vrai ou faux, adéquat ou inadéquat, en adoptant une attitude d'élève qui cherche à comprendre plutôt que de censeur qui juge, approuvant ou désapprouvant ${ }^{28}$. Lorsque le locuteur est absent ou mort et que le sens de son propos est obscur, la logique doit donner les règles et les principes de détermination du vrai sens de la phrase obscure. Quelle différence alors entre sensus genuinus et sensus verus? C'est que le sensus genuinus ne prend en compte que ce que l'auteur a voulu dire tandis que le sensus verus prend aussi en compte la relation du discours à la chose visée. Enfin la vérité (veritas) est le rapport de conformité du discours à la chose dont elle parle.

Puisque le discours externe est fait de mots, pour en percevoir le sens, il faut d'abord rechercher la signification des mots, et parce que la signification et le sens consistent dans l'accord des mots et du discours avec les choses signifiées et avec la pensée de l'auteur, il faut examiner aussi qui est l'auteur, le but du locuteur, la chose dont il est parlé, et les autres circonstances du discours. ${ }^{29}$

24. Le signe est ce qui représente quelque chose à la puissance du connaissant c'est-à-dire au sens ou à l'entendement : ainsi l'idée (dans une signification plus générale) ou le concept d'une chose quelconque dans l'âme, représente la chose à l'entendement.

25. III, vii, $\$ 36$.

26. II, viii, $\$ 52$. Clauberg nie l'existence du vide au sens des physiciens.

27 . II vII, $\$ 45$. la justice est la volonté constante et perpétuelle de rendre à chacun le sien.

28. Nempe ut prius intelligamus ea quae proponuntur quam approbemus aut improbemus III i, $\$ 3$.

29. Cum sermo externus constet ex vocabulis, ut sensus ejus percipiatur, prius vocabulorum significatio indaganda, et quia significatio et sensus consistunt in sermonis et verborum 
On remarquera ici que le sens est déterminé comme un rapport complexe qui articule deux types de rapports : a/ le rapport intentionnel du locuteur à ce qu'il veut dire ou à l'effet qu'il veut produire (et qui tient compte des capacités de la langue dont il parle et de ses propres aptitudes rhétoriques) et b/ un rapport entre le discours et la chose dont on parle qui implique à la fois la pertinence du discours et sa vérité. L'art d'interpréter c'est l'art de connaître le sens vrai d'une parole de quelqu'un avant de chercher si ce qu'il dit est vrai ou faux. Mais il n'est pas toujours facile de séparer aussi nettement sens vrai et vérité. Le problème se complique évidemment selon qu'il s'agit d'un livre divin ou humain et, dans ce dernier cas, d'un livre dont l'auteur est douteux ou reconnu.

La première connaissance requise pour comprendre un discours est celle de son auteur - cause efficiente — , de sa complexion (ingenium) et de ses positions (sententiae) habituelles. L'auteur parle-t-il lui-même ou recourtil à un porte parole ${ }^{30}$ ? Le but que s'est proposé l'auteur se voit à partir du titre ou de la préface ${ }^{31}$. Pour dégager le sens authentique et le sens vrai, le bon interprète s'aidera d'outils ou de sciences annexes ou connexes à la logique comme les dictionnaires ${ }^{32}$, la grammaire ${ }^{33}$ ou la rhétorique ${ }^{34}$. Mais il faut toujours préférer le sens propre sauf s'il présente une contradiction manifeste avec le reste du discours ${ }^{35}$.

Clauberg consacre de longs développements ${ }^{36}$, à l'analyse des modi significandi ${ }^{37}$ en insistant sur les modalités particulières d'application des

cum rebus significatis et cum mente authoris congruentia, spectandus quoque est author ipse et scopus loquentis et res de qua loquitur ; aliaeque sermonis circumstantiae. III, i $\$ 5$.

30. Quis loquitur? Deus an homo? principalis author an minister et amanuensis? III, iii, $\$ 13$.

31. "Et celui qui s'est proposé de démontrer l'existence de Dieu dans le titre de son livre ou dans sa préface, ne doit pas être estimé athée même s'il rapporte les arguments des athées ». III, iv, 19. Allusion probable à la polémique contre Descartes.

32. Lexica III, v, $\$ 27$ qui distingue entre homonymes, synonymes, et donne accès à l'étymologie.

33. Grammatica, III, v, $\$ 28$ notamment pour déterminer la signification du nombre, singuler ou pluriel quand l'ES parle de Dieu : Sic Theologi mysterium SS Trinitatis e Sacris Literis probaturi attendunt, an Deus de se in singulari an in plurali numero loquatur. Ce sont là des exemples traditionnels de l'exégèse que Spinoza reprend dans le TTP.

34. III, v, $\$ 29$ La rhétorique enseigne les tropes et donc à mieux distinguer entre sens propre et sens figuré. Clauberg cite l'exemple fort disputé parmi les théologiens, du sens propre ou du sens figuré de l'expression hoc est corpus meum. L'exemple donnera lieu dans la Logique de Port royal ( I, ch.XV) à une analyse capitale du déictique.

35. Les exemples illustratifs proposés sont aussi très classiques; "Hérode est un renard " pour dire sa méchanceté rusée ; "le bras de Dieu » pour signifier sa puissance.

36. III, ch. VII-XI.

37. En reprenant et expliquant le vocabulaire scolastique. Par exemple l'acception catégorématique ou syncatégorématique (\$37-38) ; le sens collectif ou distributif $(\$ 39)$; la signification étendue ou restreinte $(\mathbb{\$} 43)$; l'acte signifié ou exercé $(\mathbb{4} 47)$; le sens conjoint ou divisé $(\$ 48$ $49)$, formel ou identique $(\$ 50)$. 
règles proposées au cas de l'Écriture Sainte. Par exemple la règle : «Ne verba alicujus orationis facile restringas si latiorem eis significationem profunda authoris sapientia concedat ${ }^{38}$, Ne prends pas facilement les mots d'une phrase en un sens restreint si la profonde sagesse de l'auteur autorise une signification plus large » vaut principalement s'il s'agit de l'auteur divin. Ce qui doit nous conduire à nous interroger sur le statut de l'Écriture Sainte dans ce livre de logique.

\subsubsection{Spécificité de l'Écriture Sainte}

Bien que Clauberg ne se présente jamais comme un exégète et que l'Écriture Sainte ne constitue, semble-t-il, qu'un réservoir de cas parmi d'autres, le nombre très élevé d'exemples qui lui sont empruntés - bien supérieur aux exemples philosophiques ou littéraires antiques - conduit à se demander si Clauberg n'entend pas jouer un rôle décisif, un rôle de conciliateur, dans cette arène théologique ${ }^{39}$ où l'on manie la plume comme un glaive et où l'on détruit des réputations et parfois même la vie, pour des erreurs vite tenues pour des crimes $^{40}$.

D'une manière générale, le meilleur interprète est toujours l'auteur et jamais le critique : Optimus interpres verborum quisque suorum ${ }^{41}$; c'est donc à partir de ses autres écrits ou de ses faits et gestes ${ }^{42}$ qu'on cherchera des clés éventuelles pour l'interprétation. Il y a là, de la part du critique, un principe de modestie herménentique qui exclut que les Modernes comprennent les Anciens mieux qu'ils ne se sont compris eux-mêmes et un principe de générosité herméneutique ${ }^{43}$ car l'interprétation doit aussi être bienveillante et non malveillante, faire crédit au texte ${ }^{44}$. On peut même dégager toute une hiérarchie des interprètes ${ }^{45}$ qui va du meilleur (l'auteur) au pire (le calomnia-

38. III, VII, $\$ 43$.

39. Image plusieurs fois employée en II, xvi $\mathbb{1 1 8}$ et en III, xiii, $\$ 89$, opposée au silence et à la sérénité des mathématiciens. La même image est longuement reprise par L. Meyer dans la préface de l'Interpres.

40. Clauberg donne notamment (III, $x, \$ 67$ ) l'exemple de ceux qui, ne trouvant pas d'arguments décisifs pour réfuter les Sociniens, sont tenus pour sociniens alors qu'ils ne sont nullement antitrinitaires et ne professent pas les doctrines sociniennes.

41. III, ix, $\$ 51$.

42. Notamment dans le cas de phrases ironiques ou bien quand le Christ montre à Thomas ses plaies. III, $\mathrm{x}, \$ 68$.

43. Cela se marque aussi dans le vocabulaire employé : dans l'expression bonus interpres vs calumniator, bonus ne signifie pas seulement compétent (peritus) mais bien aussi bienveillant à la différence du malveillant calomniateur. cf. III, ix, $\$ 58-59$.

44. Clauberg ajoute $(\$ 63-66)$ un certain nombre de règles : chercher à concilier les thèses divergentes ; préférer la thèse postérieure à l'antérieure car la sagesse vient avec la maturité ; préférer la thèse didactique à l'argument réfutatif, l'acroamatique à l'exotérique.

45. L'interprète lui-même prend place dans une série qui comprend les commentator, interpres, correctus, criticus. III, xi, $\mathbb{\$} 71$. 
teur, ce qui est aussi un des noms du diable) en passant par le bon interprète et l'ignorant qui se trompe de bonne foi.

L'Écriture Sainte pose toutefois un cas très particulier : il s'agit bien d'un texte, écrit dans une langue donnée et qui, en tant que tel, semble relever de l'analyse herméneutique. Mais, parce que son auteur est Dieu (qui est la vérité $m \hat{e ̂ m e}^{46}$ ), un auteur dont on ne peut connaître le tempérament, la visée, le référent et les circonstances du discours que par ce seul texte, sa compréhension relève de l'analyse logique proprement dite qui examine la cohérence et la force probatoire de son propos ${ }^{47}$. Or, contrairement à ce qu'il annonce, Clauberg ne donne qu'une règle, au demeurant fort classique, spécifique à l'Écriture Sainte : " admettre un double sens si cela ne contredit pas l'analogie de la foi ». Les références à l'Écriture, très abondantes dans la III $^{\mathrm{e}}$ partie, disparaissent quasiment de la quatrième qui traite des causes d'erreurs afférentes à la formation populaire du langage, des processus de causalité et de la hiérarchie des causes, des diverses sortes de division et de définitions et de la manière dont il convient de répondre à des adversaires. Clauberg s'arrête donc juste au seuil de l'épineuse question qui divisera L. Meyer et Spinoza et tant d'autres, celle de savoir si, dans le cas très particulier de l'Écriture Sainte, on peut ou non séparer sens vrai et vérité. Autrement dit, peut-on trouver, dans un texte de l'Écriture Sainte, un sens vrai qui corresponde à quelque chose de manifestement faux ${ }^{48}$ ? Si oui, comment faut-il le traiter ? Mais avant d'en venir là, on peut dégager les enjeux pratiques de cette conception de la logique.

\subsection{Les enjeux pratiques de cette conception}

Cette logique, on l'aura compris, ne ressemble guère aux nôtres. On y rencontre des préceptes pédagogiques (il vaut mieux travailler le matin que l'aprèsmidi, l'attention y est meilleure ${ }^{49}$ ), des règles éthiques (prendre toujours le discours d'autrui d'abord dans un bon sens appartient au respect dû), des principes exégétiques (le sens propre est toujours préférable en première lecture ${ }^{50}$ ),

46. Dieu est summe verax donc celui à qui l'on doit attribuer le maximum d'autorité, prima et antiquissima veritas (IV, xi, $\$ 97$ de l'édition de 1654 ; disparaît dans l'édition de 1658).

47. Ad necessitatem hujus Analyticae uberius demonstrandam hoc etiam facit, quod verba Dei secundum eam resolvi debeant quae cum non ab homine, nec secundum humanae logicae modulum semper composita sint, manifestum est hic singularibus quibusdam monitis Analyticis opus esse. IV, i, $\$ 6$ avec renvoi au $\$ 87$ qui traite du discours décousu du Christ, style logiquement critiquable (portiones doctrinae male junctas, sine dependentia aliarum ab aliis, sine catenatione) mais adapté à la pensée des auditeurs que le Christ connaissait immédiatement.

48. Spinoza donne l'exemple des architectes de Salomon qui estimaient que le périmètre $\mathrm{du}$ cercle $=3$ diamètres alors que la mesure exacte $=$ diamètre $\mathrm{x} 3,14$.

49. I, ii, 26 aurora musis amica. Voir aussi l'éloge de l'enseignement en II, i, $\mathbb{1} 1$ : " Il n’y a rien de meilleur ni de plus désirable que d'imiter en quelque façon le Père des Lumières en éclairant l'esprit d'autrui ".

50. III, v, $\$ 30$. Application de la règle dite d'Augustin. 
des thèses théologiques (les défauts des langues naturelles sont un effet induit du péché originel ${ }^{51}$ ) et même, à l'occasion, des réflexions politiques (le peuple ne peut pas transférer au Prince plus de droit qu'il n'en possède $\left.{ }^{52}\right)$. La logique herméneutique, sous sa double forme génétique et analytique est donc une discipline architectonique qui a des applications dans tous les domaines.

Cette intrication du logique et du pratique, de la connaissance de soi et de la connaissance de Dieu, mêle à la fois des thèmes issus de l'héritage platonicien et néoplatonicien : se connaître soi-même, c'est connaître son esprit, la Logique est une medicina mentis - et un héritage cartésien : l'esprit est plus aisé à connaître que le corps ; les maladies de l'âme sont la précipitation et la prévention. La rectitude du jugement commande la rectitude de l'agir.

\section{L. Meyer et Spinoza}

Spinoza connaît Clauberg de première main. Il possède dans sa bibliothèque la Defensio cartesiana de 1652 et la Logica vetus et nova dans sa première édition de $1654^{53}$. Il ne partage pourtant pas le souci de concilier les Anciens et les Modernes puisqu'il énonce au contraire clairement son refus de l'aristotélisme ${ }^{54}$. Mais il ne peut pas être indifférent à cet auteur, ne serait-ce que parce que L. Meyer s'appuie abondamment sur lui pour défendre la distinction entre sens vrai et vérité, distinction à laquelle il fait jouer un rôle fort différent de celui que lui attribue Spinoza dans l'interprétation de l'Ecriture.

\subsection{Meyer et l'Interpres}

Meyer cite Clauberg à plusieurs reprises ${ }^{55}$, et toujours pour appuyer sa distinction du sens vrai et de la vérité. Comme lui, il rappelle qu'un texte peut comporter de multiples sens, plus ou moins expressément visés par l'auteur et qu'il faut faire crédit à l'Écriture de cette multiplicité de sens possibles visés. Mais la différence majeure avec Clauberg et Spinoza tient d'une part au statut des auteurs du texte sacré et d'autre part à celui de la norme de leur interprétation. L. Meyer ne fait pas dans la nuance : il recherche une interprétation infaillible, une norme absolument certaine, pour un texte infailliblement

51. Prol. de l'édition de 1654, $\$ 36$. Sur les implications théologiques des règles logiques, voir encore IV, xi, 98 sur les rapports entre raison et révélation (pour savoir quand il faut croire il faut d'abord percevoir clairement que l'objet de la foi a bien été révélé par Dieu) ou III, v, 29 sur les trois façons d'interpréter hoc est corpus meum qui correspondent aux trois grandes Églises chrétiennes. Clauberg y revient en IV, ii, $\$ 17$ pour justifier par le sursum corda l'interprétation figurée de la phrase.

52. IV, xiii, $\$ 120$ Application, au cas du droit et de la politique du contrat, de la règle causa nibil largitur effecto quod non habet.

53. Respectivement $n^{\circ} 28$ et 29 du catalogue.

54. "Nous n'avons cure du fatras de distinctions des péripatéticiens ». CM II, 5.

55. Interpres I, $\mathbb{} 2$ où il reprend la définition de l'interprétation; IV, $\mathbb{9}$ pour la distinction des multiples sens présents dans l’Écriture. 
vrai $^{56}$ dont l'auteur vérace - Dieu — a écrit un texte parfaitement clair à ceux qui le comprennent, c'est-à-dire finalement dépourvu de toute ambiguïté.

Le mot est pour Meyer le signe non pas de la chose mais du concept ${ }^{57}$; le concept est la chose dans l'intellect, le mot le concept proféré et par là même, la chose dans le langage. Le mot a une signification et la question du sens ne commence à se poser qu'au niveau de la phrase ${ }^{58}$. Mais le statut de simple "porte-plumes " ${ }^{59}$ concédé aux rédacteurs du texte biblique dont Dieu est l'unique auteur fait que, pour Meyer, l'Écriture, obscure aux ignorants, est parfaitement claire et sans ambiguïté pour les savants. La différence entre sens simplement dit (sensus simpliciter dictus, sens littéral), sens vrai ou visé par l'auteur, et vérité est partiellement abolie aussitôt que posée. Particulièrement lorsque le locuteur est le Christ. Quand Moïse dit « le bras de Dieu " il peut s'agir d'une métaphore pour signifier sa puissance ${ }^{60}$, mais quand le Christ dit « je suis le chemin, la vérité et la vie » ou bien " ceci est mon corps ${ }^{61}$, comment faut-il le comprendre ? Non pas au sens littéral ${ }^{62}$ mais en un sens double. Mais comment savoir qu'ici le sens propre ne convient pas ? Parce que, dans le cas de la Bible, le sens vrai (qui peut différer du sens littéral) s'accorde toujours à la vérité. Et là se marque le caractère absolu de la conception meyérienne du texte sacré et la différence avec Spinoza.

Il n'en va pas exactement de même pour les écrits profanes et humains et pour les écrits sacrés et divins. Dans ceux-ci les vérités et les vrais sens sont unis partout d'un lien indissoluble qu'on ne trouve pas dans ceux-là. Qui donc en aura extrait les vérités aura aussi extrait les sens vrais et qui aura démontré que les explications des autres contiennent des faussetés aura aussi démontré qu'elles sont fausses. ${ }^{63}$

Toute la démarche de Meyer repose sur le statut du texte sacré. Qu'il soit sacré le met absolument à part de tous les autres textes. Ce caractère sacré est un absolu et on ne doit pas en débattre puisque "le premier de tous les énoncés de théologie à savoir que Les livres de l'Ancien et du Nouveau Testament sont le Verbe infaillible du Dieu très bon et très puissant " a un

56. Int. IV $\$ 10$.

57. Int. I, $\$ 4$ ce qui laisse supposer, mais moins nettement que chez Clauberg, l'existence d'un discours intérieur, expression prélinguistique de la pensée.

58. Puis de la période et du discours.

59. Amanuenses. Interpres, IV \$5.

60. Donc ici le sens vrai n'est pas le sens littéral ou simplement dit.

61. Tous ces exemples apparaissent au ch. III, $\$ 4$, p. 47 de la trad. fr.

62. Ce qui serait ici la position catholique, qualifiée de folle : « ...et quand le Christ a dit : "ceci est mon corps", “je suis le chemin, la vérité et la vie”, chacun sait ce que ces mots désignent dans l'usage commun du langage et peut construire un sens en s'appuyant sur leur signification. Mais que ce soit le sens vrai et conforme à l'intention de l'auteur, aucun théologien sain d'esprit ne le dirait. D'où il apparaît que ces formules et donc la phrase admettent un sens double ». III, $\$ 4$, p. 47.

63. Int. IV, $\$ 4$, p. 92-93. 
statut de principe dont il n'est pas permis de discuter ${ }^{64}$. C'est là que se situe la fracture entre une lecture historique de la Bible et une lecture dogmatique. Pour Spinoza le caractère sacré du livre sacré tient à son usage :

On appelle sacré et divin ce qui est destiné à la pratique de la piété et de la religion; cela sera sacré aussi longtemps que des hommes s'en serviront religieusement; si ces hommes cessent d'être pieux, cela cessera en même temps d'être sacré ; s'ils le consacrent à des actions impies, alors ce qui était auparavant sacré devient impur et profane. ${ }^{65}$

Ainsi la Bible est un texte sacré tant qu'elle incite les hommes à l'obéissance à la règle de justice et de charité. Dès lors qu'elle nourrit la haine, elle n'est plus que «du papier et de l'encre noire en place de la parole de Dieu » ${ }^{66}$.

À partir de là tout diverge :

Pour Meyer, bien qu'il soit impossible de faire appel aux rédacteurs pour savoir ce qu'ils ont exactement voulu dire, le sens visé peut être atteint a tergo à partir de la vérité. Dieu omniscient parle directement dans l'Écriture ${ }^{67}$; donc toute vérité est ipso facto sens vrai. Il n'est pas besoin d'une méthode philologique et historique pour dégager le sens; la méthode scientifique qui nous donne la vérité sur une chose nous donne en même temps le sens vrai du discours sacré qui parle de cette même chose. Aussi, bien que Meyer soit un excellent philologue et grammairien ${ }^{68}$ et un fin connaisseur de la rhétorique, il ne présente pas d'analyse de détail de formules de l'Écriture ${ }^{69}$ - mais il développe à l'envi sa thèse de la philosophie comme norme unique et infaillible de l'Écriture Sainte en s'appuyant à la fois sur un raisonnement assez simple, pour ne pas dire fruste $\mathrm{e}^{70}$, et sur un grand nombre d'autorités, patristiques ${ }^{71}$ ou modernes. Ce qui conduit à défendre une conception complètement anhistorique de l'Écriture Sainte et de son interprétation. C'est contre cette confusion

64. Prologue p. 27 avec référence à Aristote Métaphysique 4 on ne doit pas discuter avec celui qui nie les principes.

65. TTP XII, $\$ 5$ G III 160, OS III 433

66. TTP XII, $\$ 3$, G III 159 , OS III 431.

67. «Pour bien voir que toutes les vérités des Écritures sont aussi des sens vrais, il faut d'abord considérer deux choses concernant Dieu en tant qu'auteur des livres saints : la première est qu'il est omniscient ; il connaissait très bien toutes les significations tant vraies que fausses des phrases qu'il prononçait. Non seulement il savait d'avance qu'elles pouvaient se présenter à l'esprit des lecteurs et auditeurs mais même il a prévu qu'il en serait ainsi ; et puisque rien n'arrive hors de sa providence, il a fait en sorte que les vraies se présentassent à l'esprit. » Int. IV, $\$ 8$, p. 95 .

68. Il est l'auteur d'un Dictionnaire néerlandais de renom.

69. Encore qu'il sache faire à l'occasion des remarques pertinentes sur des hébraïsmes tels que « le roi des rois » (Int. III, 21, p. 69) pour désigner le plus grand des rois (Salomon), exemple repris par Spinoza.

70. Dieu omniscient et vérace a prévu tous les sens possibles et fait en sorte que la vérité de la chose corresponde au sens vrai du texte.

71. Justin, Jérôme, Augustin, Hilaire de Poitiers. 
finale du sens vrai et de la vérité que va s'élever Spinoza en développant, lui, une méthode rigoureuse et historique d'exégèse.

La méthode pour interpréter l'Écriture ne relève pas chez Spinoza, de la logique - qui détermine la méthode pour perfectionner l'entendement ${ }^{72}-$ mais se construit selon un parallèle très strict avec la méthode d'investigation de la nature, comme il l'explique au ch. VII du TTP. Je ne reviendrai pas sur ce point qui a été fort précisément et fort bien étudié. ${ }^{73}$. On pourrait aussi établir un parallèle entre les deux conceptions de la méthode, notamment en ce qui concerne l'art de conduire son esprit tel qu'il est développé dans le TIE avec ses implications thérapeutiques, pédagogiques et politiques ${ }^{74}$ ou la relation intrinsèque entre découverte de la vraie méthode et « connaissance de l'entendement pur, de sa vraie nature et de ses lois $»^{75}$. Je voudrais plutôt m'arrêter sur le rôle que Spinoza fait jouer dans le Traité Théologico-politique à cette différence entre sens et vérité.

\subsection{La différence sens / vérité chez Spinoza}

Le sens des mots, leur univocité ou leur polysémie dépend de l'usage qui en est le maître ${ }^{76}$ et Spinoza traçant l'histoire des divers sens du mot vrai ${ }^{77}$ rejoint des remarques de Clauberg sur le même sujet ${ }^{78}:$ de la propriété d'un récit accordé avec l'évènement raconté, on est passé à l'accord de l'idée avec son objet puis à l'authenticité de l'apparence (or vrai, or faux). Le sens des mots, quoique arbitraire, ne peut être modifié volontairement :

Personne n'a jamais eu intérêt à modifier la signification d'un mot alors qu'on a souvent pu avoir intérêt à modifier le sens d'une phrase. En outre changer le sens d'un mot est chose fort difficile: qui s'efforcerait de modifier la signification d'un mot serait en même temps contraint d'expliquer selon la complexion ou la pensée de chacun d'entre eux tous les auteurs qui ont écrit en cette langue et se sont servis de ce mot dans sa signification reçue ou de les déformer avec les plus grandes précautions ${ }^{79}$.

72. «Quomodo et qua via debeat intellectus perfici ad logicam spectat » Ethique V, préf. G II 277.

73. Voir S. Zac Spinoza et l'interprétation de l'Écriture, PUF, Paris, 1965 ; A. Matheron Le Christ et le salut des ignorants chez Spinoza, Aubier Montaigne, Paris, 1971 ; J. Lagrée « Le thème des deux livres de la Nature et de l'Ecriture ». L'Écriture sainte au temps de Spinoza et dans le système spinoziste, 1992.

74. Ce que j’ai tâché de faire dans «Clauberg et Spinoza, De la logique novantique à la puissance de l'idée vraie » Méthode et métaphysique, Paris, PUPS, 1989.

75. Ep 37 à Bouwmeester, Ap. IV 251.

76. TIE $\$ 88$ où Spinoza reprend des remarques déjà longuement développées par Hobbes ou par Clauberg sur les incohérences nées du fait que les mots ont été formés arbitrairement par la foule ignorante ce qui fait que les réalités les plus positives, telles que l'infini, sont souvent exprimées par des termes à préfixe négatif.

77. CM I, 6 ; Ap. I, 352.

78. Ontosophia IX Verum et falsum, $\mathbb{\$} \$ 152-159$, pp. 307-309.

79. TTP VII $\$ 9$ G III 105 , OS III 295. 


\subsubsection{Le statut de l'Écriture Sainte et la méthode d'interprétation}

Pour Spinoza, la divinité de l'Écriture Sainte ne va pas absolument de soi ; elle doit être justifiée : "Bien plus si, sans préjugés, nous voulons attester la divinité de l'Écriture, c'est par elle seule qu'il nous faut établir qu'elle dispense les vrais enseignements moraux, cela seul en effet, nous permet de démontrer sa divinité ". ${ }^{80}$ C'est du seul fait qu'elle enseigne la vertu vraie qu'on peut démontrer cette divinité du texte sacré sans recourir à un cercle reposant sur un préjugé ininterrogé.

Mais si Spinoza reconnaît que l'Écriture est pour une grande part obscure, il ne propose pas une norme d'interprétation (sinon celle de la Scriptura sola) mais une méthode dont il fournit des échantillons qui permettent d'en découvrir le sens à partir de son histoire, sur le modèle de l'interprétation scientifique des phénomènes naturels, puisque la Bible aussi est un être naturel.

Cette interprétation vraie procède $1 /$ à partir de la connaissance des langues originales (hébreu), 2/ à partir du contexte linguistique, 3/ à partir des circumstantiae $^{81}$ : tempérament (différence des prophètes joyeux ou tristes), vie, mœurs et préoccupations de l'auteur, occasion et destinataires ${ }^{82}$, contexte évenementiel (paix ou guerre, domination ou soumission), visée, langue de rédaction, fortune du livre ${ }^{83}$ (réception, variantes, intégration ou non dans un canon).

La clarté de la phrase scripturaire peut renvoyer à l'obscurité de la vérité correspondante mais cela relève de deux démarches différentes : philologie et histoire donnent accès au sens propre et par là au sens vrai mais non pas à la vérité. La science donne la vérité mais sur des sujets qui ne sont pas explicitement l'objet de la visée de l'Écriture Sainte .

Ainsi Spinoza opère-t-il une séparation nette entre sens vrai et vérité :

J'appelle ici claires ou obscures les affirmations dont le sens se tire facilement ou difficilement du contexte de la phrase mais non pas celles dont la vérité est perçue facilement ou difficilement par la raison. Car nous nous occupons seulement du sens des phrases et non pas de leur vérité. [...] Mais pour ne pas confondre le sens vrai avec la vérité de la chose, il faut recourir dans sa recherche au seul usage de la langue ou à une argumentation qui n'admette pour fondement que l'Écriture ${ }^{84}$.

Par exemple « Dieu est un feu » est une affirmation parfaitement claire quoique fausse, et la comparaison des diverses affirmations de Moïse sur Dieu

80. TTP VII, $\$ 4$, G III 99, OS III 281.

81. TTP VII $\$ 5$ G III 101-102, OS III 285-289. On retrouve ici des remarques déjà faites par Clauberg (Logica III 5) et Meyer (Interpres III 7).

82. Par exemple pour comprendre la différence entre les exhortations du Christ dans le sermon sur la montagne, l'enseignement de Jérémie (prophète de malheur) et celui de Moïse (législateur de la loi du talion) TTP VII $\$ 7$ G III 103, OS III 291.

83. TTP VII $\$ 15$ G III 109-110, OS III 305-307.

84. TTP VII $\$ 5$, G III 100, OS III 285. 
font clairement voir que le mot « feu » doit être interprété ici en un sens métaphorique, équivalent à " Dieu est jaloux ", bien que cette deuxième affirmation soit aussi fausse que la première où " feu » était pris en son sens littéral.

De cette enquête et de cette comparaison se dégagera la différence entre la Bible comme monument historique, archive, dont certains éléments sont irrémédiablement perdus ou indéchiffrables ${ }^{85}$ (par exemple le sens de certains mots) et de son noyau éthique et rationnel qui atteste sa divinité : son enseignement moral constant de la loi de justice et de charité et l'affirmation que l'obéissance à cette loi suffit au salut. Ce noyau éthique est compréhensible par tous et chacun peut le percevoir y compris dans les traductions de la Bible car il est écrit en termes simples et fait appel à des expériences de vie commune aisément transposables :

Les enseignements de vraie piété sont exprimés dans les termes les plus courants, car ils sont tout à fait communs et tout aussi simples et faciles à comprendre. Et puisque le salut et la béatitude véritables consistent dans la vraie paix de l'âme et que nous ne nous reposons véritablement qu'en ce que nous comprenons très clairement, il s'ensuit avec beaucoup d'évidence que nos pouvons comprendre avec certitude la pensée de l'Écriture touchant les choses nécessaires au salut et à la béatitude. ${ }^{86}$

Avec Clauberg et Meyer et contre certains réformés, Spinoza prétend que la lumière naturelle ou la raison suffit au déchiffrement de l'Écriture et à la pleine compréhension de ce qu'il y a à comprendre. Elle ne suffit pas à tout comprendre mais ce qui demeure obscur n'importe pas à son lecteur en tant que lecteur de la Bible. Il n'est pas besoin d'établir la vérité de la chose, le sens vrai suffit dès lors qu'on ne cherche plus dans la Bible des enseignement spéculatifs (par ex sur la création ou l'éternité du monde ${ }^{87}$ ) mais seulement une voie de salut offerte à tous.

\subsubsection{Le rapport au vrai ne peut être solitaire}

Alors que, sans l'avoir probablement voulu, l'option meyérienne pour la science comme clé du chiffre de l'Écriture conduisait à une conception élitiste de la lecture et à la formation d'une nouvelle caste de clercs, Spinoza partage avec Clauberg l'analyse de la formation du langage : sur l'origine populaire et ignorante des mots, sur l'impossibilité de changer à volonté leur sens, sur l'adaptation du langage, ce qui le conduit à une position plus réaliste et plus féconde : il faut " parler comme le peuple sans pour autant penser comme

85. «Presque tous les noms de fruits, d'oiseaux, de poissons et beaucoup d'autres ont péri sous les coups du temps. En outre, la signification de nombreux noms et verbes de la Bible est soit complètement ignorée, soit sujette à dispute. [...] Les tours de phrase et les manières de dire propres à la langue hébraïque, le temps dévoreur les a presque tous effacés de la mémoire des hommes » TTP VII $\$ 11$ G III 106, OS III 299.

86. TTP VII $\$ 17$ G III 111, OS III 309.

87. Voir la critique de Maïmonide en TTP VII $\$ 20$ G III 113-14. 
lui » comme l'écrit Clauberg ${ }^{88}$ ou encore il faut adapter son discours à la compréhension de ses destinataires, le préparer à entendre la vérité ${ }^{89}$ car si la vérité requiert des conditions méthodologiques et gnoséologiques, elle suppose aussi des conditions politiques que l'homme sage ne peut mépriser : respect de la liberté de penser et de s'exprimer, développement suffisant des richesses, etc.

Spinoza sépare donc ce que Meyer confond : la recherche du sens vrai (par des méthodes d'exégèse historique) et l'approbation ou le rejet par la raison de la proposition ainsi comprise. Encore qu'une proposition manifestement fausse (par exemple la détermination de Dieu comme un souffle, une lumière, une personne dotée de sentiments d'amour, de colère ou de jalousie) peut très bien fonctionner efficacement pour inciter l'âme à la pratique de la justice et de la charité et par là même se trouver validée dans son domaine propre et celui-là seulement, à savoir la religion comme ensemble de croyances et de pratiques qui visent à faciliter et rendre constante la vertu vraie. Il faut donc distinguer entre l'ordre de la vertu et l'ordre de la vérité : la vérité se juge par la lumière naturelle et la raison, le sens authentique et vrai (genuinus et verus) par la connaissance de la langue du texte, des circonstances de sa rédaction, de sa visée et de son devenir, c'est-à-dire par des connaissances historiques. La philosophie a pour but la vérité ; la théologie (au sens spinoziste ${ }^{90}$ ) la vertu ; leurs domaines sont rigoureusement séparés et si Spinoza refuse de faire de l'une la servante de l'autre - alors que Meyer faisait de la théologie scripturaire la propédeutique à la vérité — on peut penser que l'ordre de la vérité (qui est unique) est supérieur à l'ordre de la vertu (dont la multiplicité correspond aux différentes natures des êtres ${ }^{91}$ ). Mais l'Écriture fait aussi connaître - et sans doute par une voie plus aisée que la philosophie - « que toutes choses arrivent par la puissance de l'Être souverainement parfait et son immuable décret » et c'est cette connaissance (qu'on pourrait aussi acquérir philosophiquement par la connaissance de l'universelle et constante légalité de la nature) qui donne à l'homme sa "satisfaction la plus haute et sa tranquillité d'âme $»^{92}$.

\section{Interprétation et adaptation ; générosité ou frilosité herméneutique}

Pour finir, je voudrais m'interroger sur le sens ultime de cette enquête : s'agitil seulement d'une recherche érudite et de la mise en évidence d'une source

88. Logique IV $\$ 70$.

89. TIE $\$ 17$ ad captum vulgi loqui ... quod tali modo amicas praebebunt aures ad veritatem audiendam.

90. Sens toujours positif dans le TTP où la théologie désigne la foi.

91. Voire à la diversité des conditions historiques car ce qui peut être vertueux dans une situation donnée : préférer la miséricorde à la justice — peut être condamnable dans une autre situation (l'époque de Jérémie contre celle de Moïse).

92. Ep. 21 à Blyenbergh 28 janv 1665, Ap. IV, 204. 
occultée ou ignorée de la pensée spinoziste - ce qui n'est pas dénué d'intérêt - ou bien cette analyse de l'articulation entre sens littéral, sens vrai et vérité a-t-elle des incidences plus fortes dans le débat herméneutique en général et dans l'interprétation de l'Écriture Sainte en particulier ?

Une des facettes du débat sur l'interprétation de l'Écriture Sainte au $\mathrm{XVII}{ }^{\mathrm{e}}$ siècle a porté sur le rôle de l'inspiration divine dans la rédaction et dans le déchiffrement du texte sacré. Certains protestants l'affirment (en se l'attribuant), d'autres (suivis par Spinoza) la nient (en craignant les délires enthousiastes), Meyer l'assimile purement et simplement à la certitude interne qui accompagne la perception de l'idée claire et distincte ${ }^{93}$. Or la conception tant de l'inspiration que de l'adaptation de l'Écriture Sainte a des conséquences directes sur la pertinence du texte et sur la liberté de sa lecture. Si le texte (ou son interprétation) est directement inspiré et s'il est adapté à un public déterminé (le peuple élu) alors sa lecture authentique est réservée à un cercle restreint de clercs. Réciproquement, si le texte sacré s'adapte à un public très large, voire universel, alors, elle peut être lue à divers niveaux ${ }^{94}$ et de différentes façons pourvu qu'elle justifie et facilite une pratique juste et droite.

S'il n'y a pas d'inspiration littérale, alors ce qui est intelligible à tous, c'est ce qui est univoque, universel, très simple et qui se dit de même façon en toute langue et toute culture, l'enseignement moral universel. Inversement, ce qui fait l'objet d'un propos significatif d'une époque ou d'une culture donnée requiert une interprétation qui est l'adaptation du lecteur aux modes de pensée d'un peuple ou d'un temps reculé. Si l'auteur est censé produire un texte polysémique adapté à tous, il doit être divin (et le rédacteur inspiré) ; si c'est le lecteur qui s'adapte, le texte n'est pas nécessairement inspiré mais peut faire sens pour tous. Ce qui fait la modernité de la logique d'un Clauberg, en dépit de la forme archaïque de l'exposé et de son désir impossible d'harmoniser la logique des Anciens et celle des Modernes, c'est le principe de générosité herméneutique et l'ouverture laissée à la liberté de l'interprétation à condition de respecter les principes de la logique justement, c'est-à-dire de l'art de bien penser et d'user droitement de sa raison. Il ne s'agit pas de comprendre l'auteur mieux qu'il ne s'est compris lui-même cela n'aurait d'ailleurs aucun sens si l'on admet que l'auteur en question est Dieu - mais d'admettre qu'il y a peut-être plus de sens dans le texte que ce que d'autres y ont perçu ou que mon interprétation n'est pas la seule possible, sans que pour autant le sens du texte soit tenu pour labile ou inconsistant. Contre ce que j'appellerai une pratique herméneutique frileuse qui veut qu'un seul sens vaille, celui de l'auteur ou celui du lecteur savant disant aux

93. «Puisque cette conscience nous persuade indubitablement, nous dicte, nous atteste et nous inspire que la chose perçue est vraie, c'est à juste titre et à bon droit qu'on peut l'appeler persuasion, dictée, témoignage et inspiration de Dieu ou de l'Esprit saint ». Interpres V, $\$ 6$, p. 111.

94. Historique, éthique, théologique... 
autres comment il faut lire et penser, il me paraît bon de défendre, dans certaines conditions, un principe de générosité herméneutique, incitant une pratique de lecture ouverte, réceptive aux changements des temps mais aussi à la richesse inépuisable de sens de ces grands textes, philosophiques, littéraires, sacrés, qui ont marqué notre culture et dans lesquels, j’ai envie de dire dans le milieu desquels, nous avons appris à lire, à penser et à amplifier notre expérience de vie et qui nous furent ainsi instituteurs d'humanité.

\section{Bibliographie}

Clauberg, Johannes. Opera omnia philosophica, Amsterdam 1691; reprint 2 vol. Olms, 1968. Vol 2 : Logica vetus et nova $(1654,1658)$.

Meyer, L., Philosophia S. Scripturae interpres, Exercitatio paradoxa in qua veram philosophiam infaillibilem S. Literas interpretandi normam esse apodictice demonstratur et discrepantes ab hac sententia expenduntur ac refelluntur. Eleutheropolis (Amsterdam) 1666, Trad. fr. J. Lagrée et P.-F. Moreau La philosophie interprète de l'Ecriture Sainte Paris, Intertextes 1988.

Spinoza, Baruch, Euvres complètes en quatre volumes publiées par Carl Gebhardt, Heidelberg, (réédition de 1972). Euvres en 4 vol. trad. Appuhn ; les citations du Traité Théologico-politique sont prises dans la traduction Lagrée Moreau, Paris, PUF, 1999.

Bouillier, Francisque, Histoire de la philosophie cartésienne, Paris, 1868, vol. 1, ch. 13, pp. 293-300.

Damiron, J. Philibert, Essai sur l'histoire de la philosophie en France au XVII ${ }^{e}$ siècle, 1846, reprint Genève 1970, ch.V, pp. 126-154.

Lagrée, Jacqueline « Sens et vérité. Philosophie et théologie chez L. Meyer et Spinoza »Spinoza's Early Writings, Studia spinozana 4 (1998), pp. 7591 ; "Clauberg et Spinoza, De la logique novantique à la puissance de l'idée vraie » in Méthode et métaphysique, TetD n², Paris, PUPS 1989 pp. 19-45.

Bordoli, Roberto, Ragione e Scrittura tra Descartes e Spinoza, Saggio sulla "Philosophia S. Scripturae Interpres » di Lodewijk Meyer e sulla sua recezione, Milan, Francoangeli, 1997, 472p. 